\title{
Gradient-induced voltages on 12-lead ECGs during high-duty-cycle MRI sequences and a theoretically-based method to remove them
}

\author{
Shelley H Zhang ${ }^{6 *}$, Zion T Tse ${ }^{1}$, Charles L Dumoulin² ${ }^{2}$ Ronald D Watkins ${ }^{3}$, Wei Wang ${ }^{6}$, Jay Ward ${ }^{4}$, \\ Raymond Y Kwong ${ }^{5}$, William G Stevenson 5 , Ehud J Schmidt ${ }^{6}$ \\ From 18th Annual SCMR Scientific Sessions \\ Nice, France. 4-7 February 2015
}

\section{Background}

An MRI-compatible 12-lead ECG platform, equipped with MRI-gradient induced-voltage removal hardware and magneto-hydrodynamic voltage removal software [1], was previously applied to physiological monitoring and synchronization of cardiac imaging of patients inside MRI. This approach had limited success for highduty-cycle [(total Gradient-ramp-time per R-R)/(R-R time) $>20 \%$ ] MRI sequences, such as Steady State Free Precession (SSFP), Short-TR Gradient Echo (GRE), and Short-TR Fast Spin Echo. The study objective is to measure and develop a method to remove gradient-induced voltages on 12-lead ECGs during high-duty-cycle MRI sequences.

\section{Methods}

A modification of the hardware developed in [1] enabled measuring the gradient-induced voltage over a $24 \mathrm{kHz}$ frequency-range and $+/-10 \mathrm{~V}$, together with the $\mathrm{x}, \mathrm{y}$, and $\mathrm{z}$ gradient waveforms. ECGs were measured in 9 volunteers at 3T (Siemens Skyra). A theoretical equation for the gradient-induced voltages on each ECG electrode $\left(V_{i}\right.$, where $i=1,2,3 . .9$ was derived, based on Maxwell's equations [2] and concomitant fields [3] [Fig. 1A]. It includes 1 st and 2 nd order gradient waveform terms and estimates induced voltages even on ECG electrodes positioned farthest from magnet iso-center, such as the limb leads.

The 19 equation coefficients were obtained during 6-8 second training sequences, consisting of highly accelerated (3-4sec, GRAPPA $=6-8)$ versions of each sequence,

${ }^{6}$ Radiology, Brigham and Women's Hospital, Boston, MA, USA Full list of author information is available at the end of the article followed by non-imaging segments. The non-imaging segments were used to obtain the shape of the true ECG traces over the entire R-R cycle ("template"). This template was subtracted from ECG traces acquired during imaging, resulting in the net gradient-induced voltages, which was then fit to the equation, providing the 19 coefficients for each electrode.

Multi-slice imaging was then performed, with realtime subtraction of the gradient-induced voltages from each acquired ECG trace, utilizing the computed $V_{i}$.

\section{Results}

Measured limb-lead ECG voltages during SSFP imaging, with the heart at iso-center (Fig. 1B), were 0.7-1.0 Volt PTP, with frequency components up to $20 \mathrm{KHz}$ (Fig. 1C). Applying the equation for gradient-induced voltage removal during GRE (Fig. 1D-E), and multi-slice SSFP imaging (Fig. 1F), resulted in a normalized-difference between the recovered ECG trace and the true ECG of $<20 \%$. Equation coefficients varied by subject, sequence, sequence parameters, and slice orientation (Fig. 1G).

\section{Conclusions}

An equation was derived for the strong gradientinduced voltages observed in 12-lead ECGs during highduty-cycle MRI sequences. A rapid training sequence permitted computing equation-coefficients, followed by real-time gradient-induced voltage removal during imaging.

\section{Funding}

NIH U41-RR019703, R03-EB013873-01A1, AHA 10SDG261039. 


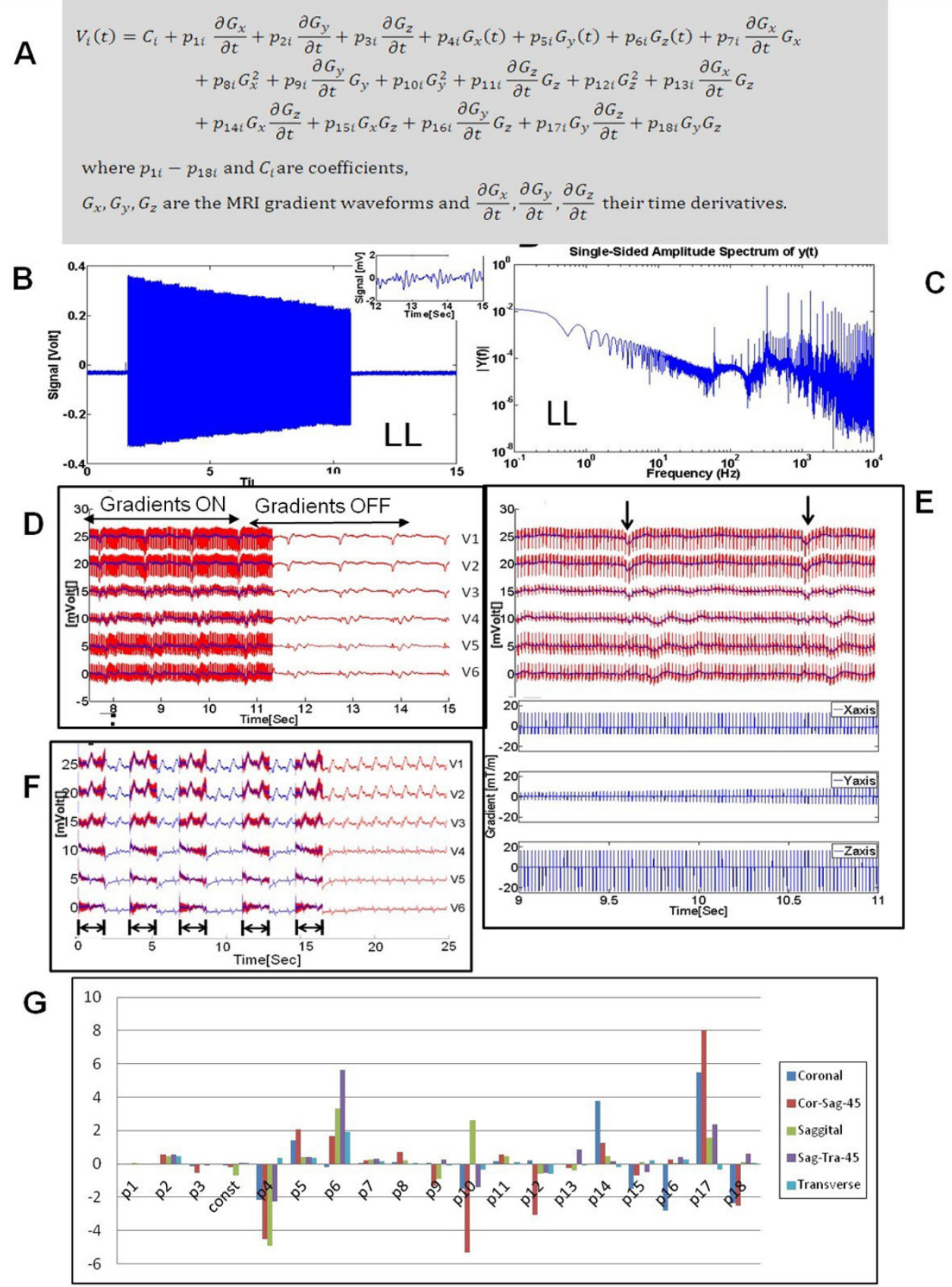

Figure 1 (A) Theoretically derived 19-parameter equation for the gradient-induced voltage at each ECG Electrode (i). (B) Measured induced voltage at the Left Leg (LL) during and SSFP sequence (TR/TE=3.09/1.32ms). Magnitude is 0.7 Volts. Inset shows real ECG when MRI was not pulsing. (C) Frequency spectrum of induced-voltage on LL during SSFP sequence. Spectral peaks are at $60 \mathrm{~Hz}$ and a harmonics of (1/TR) frequency $\sim 320 \mathrm{~Hz}$. (D) ECG traces V1-V6 recorded during a training GRE sequence (TR/TE=20/1.97ms), which consisted of intermittent imaging (Gradient ON) and non-imaging (Gradient OFF) segments. The non-imaging segment was then subtracted from the traces acquired during imaging ("corrupted ECGs", red lines), and the resulting traces fitted to the theoretical equation, in order to compute its coefficients $P_{11-} P_{18 i}$ and $C_{i}$. The calculated induced-voltages were then removed from the corrupted ECGs, resulting in restored ECGs (blue line) that were very similar to the true ECG. (E) Zoomed view of imaging-segment in (D), showing corrupted (red) and restored (blue lines) traces. Bottom Section showing gradient waveforms along $x, y$, and $z$ directions recorded during this time. Black arrows highlight QRS complexes, which are easily observed in the restored traces. (F) during a full-resolution multi-slice SSFP sequences, the recorded ECG traces (red line) from 5 parallel slices were cleaned (blue line) in real-time using the equation, using coefficients calculated from a prior single-slice accelerated (training) sequence. (G) The 19-fit parameters for the V6 electrode for the same SSFP sequence acquired along multiple directions in a single volunteer; Coronal, 45 degrees oblique between Coronal and Sagittal, Sagittal, 45 degrees oblique between Sagittal and Transverse, Transverse. 


\section{Authors' details}

${ }^{1}$ Engineering, The University of Georgia, Athens, GA, USA. ${ }^{2}$ Radiology,

Cincinnati Children's Hospital Medical Center, Cincinnati, OH, USA.

${ }^{3}$ Radiology, Stanford University, Stanford, CA, USA. ${ }^{4}$ E-Trolz InC, Andover, MA,

USA. ${ }^{5}$ Cardiology, Brigham and Women's Hospital, Boston, MA, USA.

${ }^{6}$ Radiology, Brigham and Women's Hospital, Boston, MA, USA.

Published: 3 February 2015

\section{References}

1. Tse: MRM. '13

2. Bowtell: MRM. '00

3. Bernstein: MRM. ' 89 .

doi:10.1186/1532-429X-17-S1-P243

Cite this article as: Zhang et al:: Gradient-induced voltages on 12-lead ECGs during high-duty-cycle MRI sequences and a theoretically-based method to remove them. Journal of Cardiovascular Magnetic Resonance 2015 17(Suppl 1):P243.

Submit your next manuscript to BioMed Central and take full advantage of:

- Convenient online submission

- Thorough peer review

- No space constraints or color figure charges

- Immediate publication on acceptance

- Inclusion in PubMed, CAS, Scopus and Google Scholar

- Research which is freely available for redistribution

Submit your manuscript at www.biomedcentral.com/submit 